\title{
Branchial Cyst in the Parapharyngeal Space: A Case Report
}

\author{
Iyad Said Hamadi ${ }^{a} \quad$ Lubna Lutfi $^{b} \quad$ Asma Anan Mohammed ${ }^{b} \quad$ Zahr Alkhadem $^{\mathrm{b}}$ \\ ${ }^{a}$ Ears Nose Throat Department, Dubai Hospital, Dubai Health Authority, Dubai, United Arab Emirates; ${ }^{\text {b}}$ Medical \\ Internship Program, Medical Education Department, Dubai Health Authority, Dubai, United Arab Emirates
}

\section{Keywords}

Branchial anomalies · Branchial cyst · Sinus · Fistula ·

Dysphagia

\begin{abstract}
Branchial cleft cysts are congenital anomalies that most commonly arise from a failure of fusion of the second branchial arch during embryonic life. They usually present as a swelling in the lateral side of the neck, below the mandible. In this article, we present a case of a 28-year-old female patient with a right branchial cyst measuring $7 \times 6 \times 5 \mathrm{~cm}$, who presented with an asymptomatic, rapidly growing mass in the right anterior triangle of the neck that abutted the right external carotid artery, leading to stenosis of the vessel that is preceded by dilatation above the site of compression. She underwent excision of the cystic mass with preservation of the facial nerve and presented no active complaints on follow-up a few weeks postoperatively.
\end{abstract}

(C) 2021 The Author(s)

Published by S. Karger AG, Basel
(C) 2021 The Author(s)

Published by S. Karger AG, Basel

This is an Open Access article licensed under the Creative Commons Attribution-NonCommercial-4.0 International License (CC BY-NC) (http://www.karger.com/Services/OpenAccessLicense), applicable to the online version of the article only. Usage and distribution for commercial purposes requires written permission.

\section{Introduction}

Branchial arches give rise to numerous anatomic structures in the head, face, and neck during embryonic development. Branchial malformations including cysts, fistulas, and sinuses develop from incomplete obliteration of the clefts and pouches separating each arch, most commonly arising in the 2 nd arch and making up $95 \%$ of all branchial anomalies [1]. Branchial cleft cysts are believed to be a result of failure of fusion of the second branchial arch [2], although some theories suggest that cystic degeneration of the first, second, and third branchial clefts or cystic degeneration of epithelial elements of cervical lymph nodes can also lead to cyst formation [3]. Branchial cysts are more likely to affect female than male individuals and usually present at the 3rd decade of life [4] as a nontender swelling in the lateral side of the neck inferior to the mandible. They can grow in size, particularly after an upper respiratory tract infection and sometimes display signs of inflammation and abscess formation such as pain, swelling, and edema. Acute enlargement may cause obstructive symptoms such as respiratory compromise or dysphagia. Occasionally, the cyst is associated with a sinus or a fistula related to the sternocleidomastoid muscle. Diagnosis can be confirmed

Correspondence to:

Lubna Lutfi, Lu.hussamm@live.com 


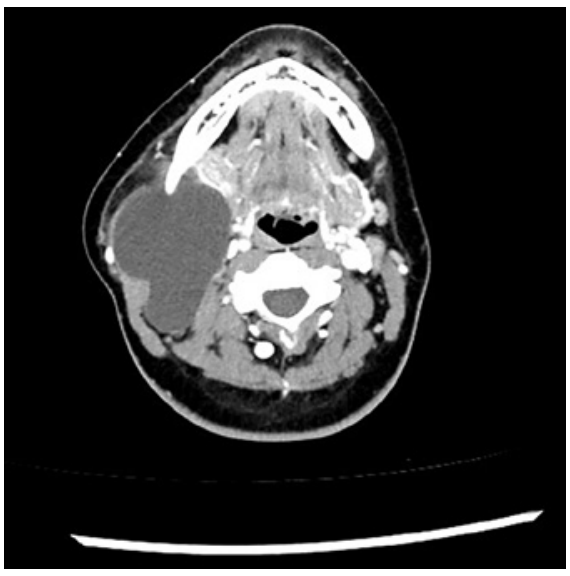

Fig. 1. Contrast-enhanced CT scan of the neck, axial view of the neck showing the mass extent and relation to adjacent structures.

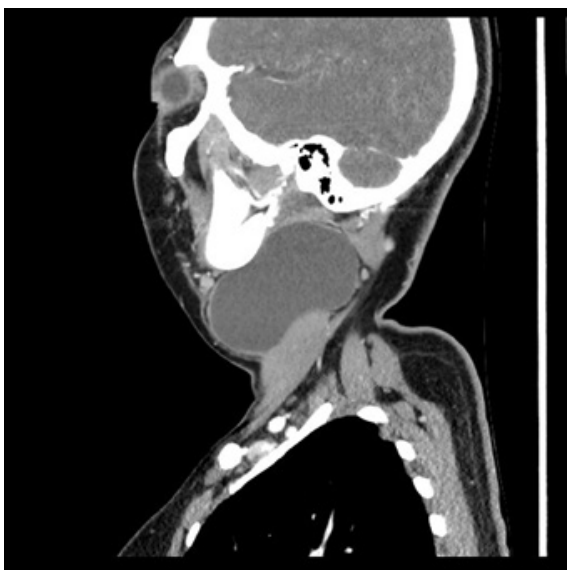

Fig. 2. Contrast-enhanced CT scan of the neck, sagittal view.

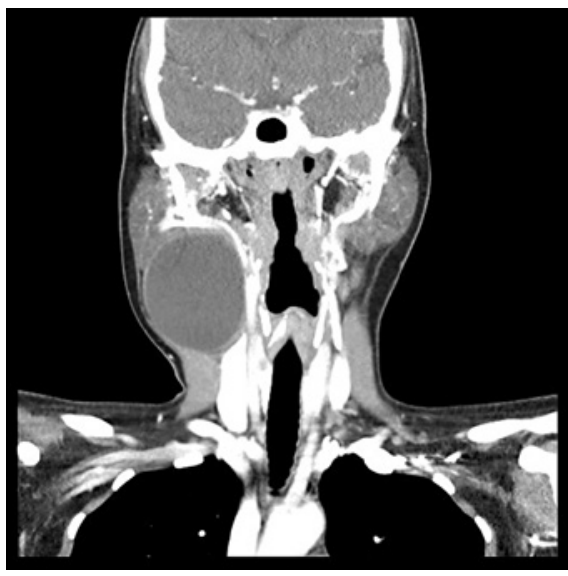

Fig. 3. Contrast-enhanced CT scan of the neck, coronal view. by fine-needle aspiration cytology and the presence of a fluid-filled cyst on imaging, that is, on a CT scan, US, or MRI. Treatment is surgical excision of the cyst, although antibiotics can be given preoperatively if the cyst is infected [5].

\section{Case Report}

A healthy 28-year-old female patient presented to the primary healthcare clinic complaining of a neck swelling that had been progressively growing in size over the last 6 months. The swelling was associated with a 1-month history of neck pain radiating to the right shoulder and right upper limb, with occasional dysphagia. She, however, did not report a change in her breathing or voice and did not experience any night sweats or unintentional changes in weight. Furthermore, this is the first episode of this nature, and past medical and surgical history is insignificant. There is also no known family history of a similar complaint.

On examination, the patient was vitally stable and looked generally well. Upon local examination, inspection revealed a visible 6 $\times 6$ - $\mathrm{cm}$ swelling in the right anterior triangle of the neck, extending up to the right submandibular angle superiorly, to the right ear posteriorly, and to the root of the neck inferiorly. When palpated, the swelling was soft, cystic, mildly tender, and nonmobile. Furthermore, it was noncompressible, nonreducible, and nonpulsatile. It was adherent to the underlying structures, with no overlying skin changes or inflammatory changes. Auscultation revealed no bruit. Examination of the local and regional lymph nodes was unremarkable.

A full blood count was done, which was unremarkable, and inflammatory markers were not elevated. A CT scan of the neck with contrast was performed, revealing a $7 \times 6 \times 5-\mathrm{cm}$ right cystic lesion consistent with a 2 nd branchial cleft cyst (shown in Fig. 1). It was related to the anteromedial aspect of the right sternocleidomastoid muscle and the posterior aspect of the right submandibular salivary gland, which was displaced anteriorly. The cyst extended to the right parapharyngeal space, pushing the pharyngeal wall and in turn narrowing the hypopharynx and upper airway (shown in Fig. 2). It also abutted the right carotid artery and right jugular vein (shown in Fig. 3).

A 3D CT scan of the head and neck was also performed to visualize the anatomy of the area surrounding the swelling (shown in Fig. 4). The 3D scan demonstrated right-sided compression of the external carotid artery by the cystic lesion, leading to stenosis followed by caudal dilatation of the vessel in contrast to the left side which showed a normal external carotid artery. Furthermore, the scan shows lateral displacement of the external jugular vein on the right side, in comparison to the normal left-sided external jugular vein (shown in Fig. 5).

The patient underwent excision of the branchial cyst 1 month after the clinic visit. The excision was performed in supine position, wherein an oblique horizontal incision was made on the anterior border of the sternocleidomastoid muscle, followed by identification and dissection of the cyst with special attention to preservation of the surrounding vital structures. The cavity was cleaned and appropriate homeostasis was achieved, followed by closure of the incision. The gross excised specimen measured $6 \times 5 \times 2 \mathrm{~cm}$ and contained brown-colored fluid. Moreover, the internal surface was smooth and spotted with white patches, and a few small lymph nodes were found attached to the edges. The postoperative course was uneventful, and the patient was discharged 2 days postoperatively.

The patient was seen in the outpatient clinic 3 weeks later for follow-up, upon which she did not report any active complaints. On examination, the wound was dry, measuring $7 \mathrm{~cm}$ in length, with well-approximated edges. However, mild numbness was elicited over the right submandibular area and external ear. Upon further examination, the right accessory, hypoglossal, and facial nerves were deemed intact and hearing remained unchanged. 


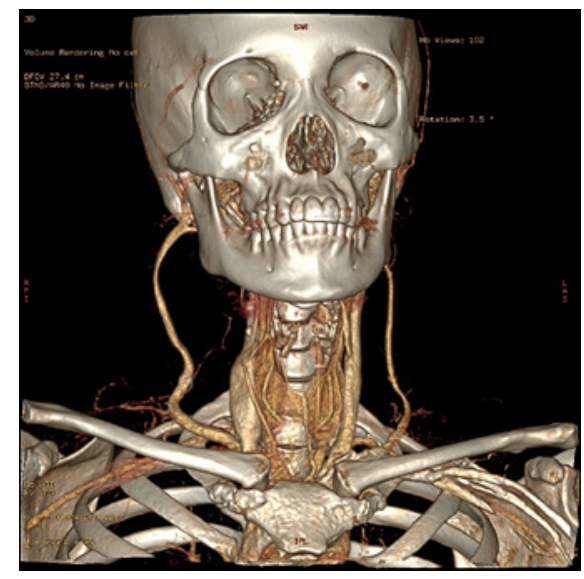

Fig. 4. 3D CT scan of the head and neck.
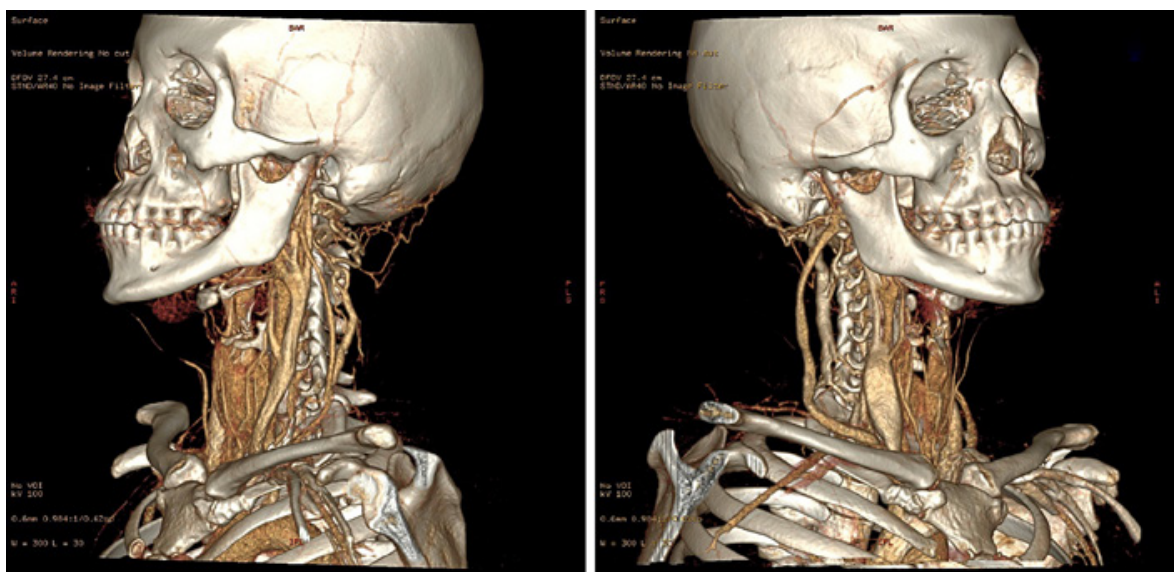

Fig. 5. 3D CT of the head and neck showing the right side of the neck. The cyst is seen compressing the external carotid artery, which is stenosed at the site of compression followed by caudal dilatation as well as the laterally displaced right external jugular vein in comparison to the left side of the neck where the vessels are in the normal anatomical position.

\section{Discussion}

Branchial arches in humans consist of 5 pairs of arches that give rise to structures in the head and neck. Each arch gives rise to specific organs. Although quite controversial, there are 4 proposed theories of origin of branchial cysts, composed of incomplete obliteration of branchial clefts and pouches, persistence of pre-cervical sinus vestiges, cystic degeneration of cervical lymph nodes, and thymopharyngeal ductal remnant. The most accepted theory to date is the failure of involution of the third and fourth arches, covered by the second arch, during development which results in trapping of epithelial cells within and development later in life to form a branchial cyst [5].

Second branchial arch anomalies are the most common branchial anomalies, making up 95\% of diagnoses. They develop more commonly in female individuals in the third decade of life, usually on the lateral side of the neck below the mandible [6]. However, they can atypically present at a later age [7]. Sometimes, they develop a sinus or a fistula communicating with the sternocleidomastoid muscle or the tonsils [5].

Clinical presentation: The history obtained from the patient typically reveals a single mass in the neck, with or without an increase in size. The patient may depict symptoms of compression such as dyspnea or dysphagia, or even snoring [8], if the mass extends to adjacent structures. It can become inflamed and tender after an upper respiratory tract infection. Physical examination usually reveals a smooth, nontender and nonfluctuant mass [9].
Differential diagnosis of the lesion may include malignant or benign lymph nodes, malignant or benign neoplasms, cervical adenitis, inflammatory lymphadenopathy, and bacterial and viral infections. Diagnosis can be confirmed by fine-needle aspirate cytology, which typically reveals stratified squamous epithelium with keratinous debris. In addition, an imaging modality such as CT scan with contrast, US of the skin and soft tissue, or MRI can be used to visualize the lesion and measure its extent [5].

Definitive treatment is surgical excision. However, infection and abscess formation can complicate the cyst; therefore, such cases warrant treatment with antibiotics prior to surgical management. Postoperative complications of surgical excision include damaging nerves and blood vessels in the neck, that is, facial nerve, hypoglossal nerve, vagus nerve, and carotid vessels [5]. Moreover, conservative surgical treatment is an option of therapy in those patients who refuse surgical excision, as reported in a case where transoral exercises were done followed by drainage of the cyst and marsupialization of the pharyngeal wall [10].

\section{Conclusion}

Although branchial cysts are typically small in size and remain asymptomatic, patients may present with atypical features or symptoms, leading to an incorrect diagnosis. It is, therefore, imperative to perform adequate imaging 
to ascertain the extent of the lesion and its relation to surrounding vital structures in the neck. In our case, because of the large size of the cyst, it resulted in compression of the right external carotid artery; this change in the normal tomography could yield further complications to the vessel. Therefore, conservative management was not an option. Surgical excision remains the definitive treatment to ensure complete eradication and prevent recurrence.

\section{Statement of Ethics}

All personally identifying information, such as name, was excluded from the study. Written informed consent was obtained from the patient for publication of this case report and any accompanying images.

\section{Conflict of Interest Statement}

The authors have no conflicts of interest to declare.

\section{Funding Sources}

There are no funding sources or sponsors for this report.

\section{Author Contributions}

I.S.H.: contributed to the conception of the case report. L.L.: contributed to the abstract and case report. A.A.M.: contributed to the introduction and conclusion. Z.A.: contributed to the discussion.

\section{References}

1 Acierno SP, Waldhausen JH. Congenital cervical cysts, sinuses and fistulae. Otolaryngol Clin North Am. 2007;40(1):161-viii.

2 Wagner A, Hansen R. Neonatal skin and skin disorders. In: Pediatrics dermatology. 2nd ed. New York, NY: Churchill Livingston; 1995. p. 3-291.

3 McLatchie G, Chikwe J, Borley NR. Head and neck surgery. In: Oxford handbook of clinical surgery. 4th ed. Oxford: Oxford University Press; 2013. p. 221-38.
4 Karande P, Ingale Y, Chavan S, Deshmukh R. Branchial cleft cyst: A case report and review of literature. J Oral Maxillofac Pathol. 2014; 18(1):150.

5 Raftery AT, Ofo E. Head, neck and otorhinolaryngology. In: Churchill's pocketbook of surgery, international edition. Place of publication not identified. Churchill Livingstone; 2016. p. 129-55.

6 Daou F. Branchial cyst: an often forgotten diagnosis. Asian J Surg. 2005;28(3):174-8.

7 McClure MJ, McKinstry CS, Stewart R, Madden M. Late presentation of branchial cyst. Ulster Med J. 1998;67(2):129.
8 Saussez S, De Maesschalk T, Mahillon V, Filleul O, Louryan S. second branchia;l cyst in the parapharyngeal space: a case report. Auris, Nasus. Larynx. 2009;36(3):376-9.

9 Hong CH. Branchial cleft cyst. Background, pathophysiology, etiology. Medscape; 2020 [cited 2020 Nov 3]. Available from: https:// emedicine.medscape.com/article/1110351overview.

10 Diaz-Manzano JA, Sanchez-Martin N, Iniesta-Alcazar J, Medina-Banegas A. conservative surgical treatment of pharyngeal branchial cyst. Auris, Nasus, Larynx. 2007;35(1):161-4. 